\title{
Der Hunger in physiologischen Lehrbüchern von Haller bis Valentin*
}

\author{
Von Erich Hintzsche
}

Dieser gelegentlich der H. E. Sigerist-Conferenz 1962 in etwas kürzerer Form vorgetragene Bericht ist rein referierend. Er beschränkt sich auf Auszüge aus physiologischen Lehrbüchern eines Jahrhunderts, nämlich auf die Zeit von Hallers Primae Lineae Physiologiae (1747) bis zur 2.Auflage von VAlentins Lehrbuch der Physiologie (1847). In dieser Zeitspanne entwickelte sich die Physiologie von der durch HALlen erneuerten Experimentalforschung durch eine Phase naturphilosophischer Betrachtungsweise zu einer mathematisch-statistisch fundierten Naturwissenschaft, als die sie uns zuerst bei G. G. VALENTIN entgegentritt.

Von wissenschaftlicher Bedeutung scheint mir in dieser Zusammenstellung die Bildung des Begriffes «Gemeingefühl» zu sein, und das nicht nur, weil dieses im Mittelpunkt des Referates steht, sondern weil sich wieder einmal zeigt, daß wir der so oft gelästerten Naturphilosophie Vorstellungen und Begriffe verdanken, ohne die auch die heutige Biologie noch nicht auskommen kann.

In seinem Grundriß der Physiologie befaßte sich Haller nicht zum ersten Male mit dem Hunger als einem physiologischen Problem; er hatte schon in seiner neuen Ausgabe von BoerhaAves Praelectiones academicae in proprias institutiones rei medicae Erläuterungen aus der Literatur zusammengetragen, wirklich eigene Ansichten vertrat er dort aber nicht. Sie finden sich zuerst in dem für den Unterricht bestimmten Grundriß. Im Kapitel XXI heißt es dort: «Der Schöpfer setzte dem Menschen einerseits den Schmerz, andrerseits das Lustgefühl zu Wächtern, jenen um Übles abzuwenden, dieses um ihn zu nützlichen Handlungen zu veranlassen» (S. 312). Dementsprechend wird auch die Aufnahme der Nahrung auf diese zwei regulierenden Faktoren zurückgeführt, denn sie wird nach HALLER (S. 313) kontrolliert

1. durch den Schmerz, den man Hunger nenne, und

2. durch das Vergnügen, das die Geschmacksempfindungen schaffen.

* Herrn Professor A.von Muralt zum 60. Geburtstag gewidmet. 
In dem Bemühen, alles erklären zu wollen, suchte HALLER auch nach den Gründen des Hungergefühles. Er schloß sich der schon früher geäußerten Meinung an, daß die Schleimhautfalten des Magens gegeneinander reiben und so die Nerven gereizt werden, wenn peristaltische Bewegungen oder Kontraktionen des Zwerchfelles oder der Bauchmuskeln stattfinden. Auf diese Weise würde der Mensch nachdrücklich vor der Gefahr gewarnt, die in einer Enthaltung von der Nahrung liege, außerdem würde er dadurch gezwungen, sich durch seine Arbeit Nahrung zu verschaffen (S. 314). Zu dem Hungergefühl trage vielleicht auch der Magensaft bei, der schärfer würde, wenn er nicht gar faule. Von den früher genannten weiteren Faktoren: verschlucktem Speichel oder Leere des Magens als Ursache des Hungergefühles spricht Haller in dem Grundriß nicht.

Ganz im gleichen Sinne, nur weit ausführlicher behandelte er dann das Thema wieder im 6. Band seiner Elementa physiologiae (1764). Er geht dabei (S. 2) von der Tatsache aus, daß der ständige Verlust des Körpers durch Sekrete und Abscheidungen Ersatz durch Zufuhr neuer Materie erfordere, sonst würde der Körper in kurzer Zeit einem Schwund unterliegen. Hunger und Durst seien die dringenden Ursachen, die uns nötigen, nicht ohne Nahrung und Getränk zu bleiben. Da HÁller sich oft wiederholt und frühere Ausführungen durch Nachträge ergänzt, folge ich seinen Angaben nicht Paragraph auf Paragraph, sondern stelle seine Ansichten nach einer eigenen Disposition zusammen:

Die Unentbehrlichkeit von Speise und Trank wird aus der göttlichen Weisheit erklärt (S. 164). Gott habe den Tieren eine bestimmte Lebensfrist gesetzt, zu deren Erreichung Nahrungszufuhr unbedingt erforderlich sei; um diese Absicht zu erreichen, verlocke er einerseits durch angenehme Geschmacksempfindungen, andererseits drohe er Mensch und Tier, die zur Trägheit geneigt seien, mit einer Strafe, «dem heftigen, grausamen und unerträglichen Schmerz des Hungers und Durstes », so daß auch der Faule für Speise und Trank sorgen muß. HaLLER bezeichnet geradezu den Hunger als «die grausame Mutter des Fleißes» (S. 2).

Hunger äußert sich nach HALLER in einer unangenehmen Empfindung (S. 182), gleichsam einer Art von ätzendem Schmerz, der seinen Sitz ausschließlich im Magen hat. Er führt zu Entkräftung und Schlaflosigkeit, die Körpersäfte werden zunehmend schärfer wegen der ungenügenden Erneuerung der Materie. Die höchst scharf gewordene Galle tritt in den Magen zurück, alle Sekrete werden an Menge geringer, der Urin riecht unangenehm, ebenso wird der Atem stinkend (Aceton bei vermehrtem Fettabbau!). Durch 
Riß kleiner Gefäße entstehen Blutungen in Magen, Darm und Nase; anfänglicher Verdrießlichkeit können Erregungszustände und Wutanfälle folgen. Schließlich tritt der Tod "wie der von einer hitzigen Krankheit» (S. 168) ein. Mit Celsus übereinstimmend stellte Haller fest, daß junge Leute den Hunger schlechter überstehen als alte, was aus dem wachstumsbedingten höheren Nahrungsbedarf erklärt wird. Wenig kritisch führt HaLler Beispiele von Hungertod nach bestimmter Zeit an; für Erwachsene rechnet er mit 7 bis 9 Tagen Überlebensdauer. Im Bestreben, vollständig zu sein, erwähnt HALler sogar einzelne Beispiele freiwilligen Hungerns mit Tod am 13., 19., ja sogar am 28. Tag; ich halte aber diese Angaben nicht für genau genug überprüft.

Sehr ausführlich sind die Kapitel über lange Fastenzeiten bei Tier und Mensch (S. 168-175). Es heißt dort: Muntere Tiere von warmem Blut ertragen den Hunger nicht so gut (S. 170). Im übrigen verweist er auf die winterschlafenden Tiere, die lange zu fasten vermögen. Nach den Befunden an lange Zeit hungernden Fischen, Amphibien und Reptilien soll zugleich mit dem Schwund des Fettes auch die Blutmenge abnehmen; hier führt Haller seine einzige eigene Experimentalbeobachtung über die Folgen des Hungerzustandes an (S. 166): Hungernde Frösche zeigten fast die Hälfte der Blutgefäße leer und nur wenige gelbliche Kügelchen darin, bei wohlgefütterten Fröschen waren dagegen die Schlag- und Blutadern mit schönstem rotem Blut angefüllt.

Übergehend zu Beispielen langen Fastens von Menschen, schreibt Halle R (S. 171): In der Medizingeschichte existieren schon von früher Zeit an Berichte über Menschen, zumeist Frauen, die Monate oder selbst ganze Jahre ohne Speise zugebracht haben. Sofern keine Betrügereien dabei vorgekommen seien, müsse man den wahren Urkunden ihre Glaubwürdigkeit zugestehen, zumal in neuerer Zeit gut beobachtete Leute wirklich lange Zeit ohne Speise, Trank und Auswürfe gelebt hätten. In einer langen Liste nennt er dann die von ihm gesammelten Beispiele mit den Belegstellen, ich greife davon nur ein paar heraus:

ein auf einem Felsen Verirrter lebte 4. Tage ohne Nahrung, ein in der Wüste verirrter Araber wurde nach fünftägigem Fasten wieder gesund, ein unter dem Schnee Vergrabener hielt 7 Tage aus, ein Mädchen, das durch eine Scherbe im Schlund am Essen verhindert war, 8 Tage; 
auch für den 9. und 10.Tag werden noch einige Beispiele aufgeführt, doch sind schon unter diesen und vor allem unter den folgenden ganz deutlich die psychisch Alterierten weit überwiegend. Auch gibt Haller bei vielen ausdrücklich an, sie hätten wenigstens Wasser zu sich genommen. Das gilt besonders für die mehrere Seiten umfassenden Beispiele von Fastenzeiten von 18 und mehr Tagen. Haller selbst faßt zusammen, welche Auslegung man solchen Berichten geben müsse (S. 175): sie betreffen meist Frauen, fast alle melancholisch oder hysterisch, einige närrisch, schlafsüchtig, unempfindlich oder wegen verstopfter Nerven gefühllos. Bei diesen könne die Seele die Reize der Natur wegen tauber Werkzeuge nicht wahrnehmen.

Weiter kommt HaLler auf die Ursachen des Hungergefühles zu sprechen (S. 183); er empfindet ganz richtig, daß langes Fasten dessen Verringerung voraussetzt, wovon in den angeführten Beispielen melancholischer und hysterischer Frauen Andeutungen zu finden sind. Wie aber ist das Fasten der Tiere z.B. im Winterschlaf möglich? Hier hilft sich Haller mit der Annahme verringerter Bewegung im Winterschlaf, so daß er doch wieder das Reiben der Magenfalten als Ursache des Hungergefühles gelten lassen kann. Ob auch das in den Falten vorhandene Blut einen «juckenden Reiz» auf die Nerven ausüben kann, vermag er nicht zu widerlegen. Ganz hypothetisch drückt er sich über den Anteil der Magensaftabsonderung am Zustandekommen des Hungergefühles aus. Bei Fastenden werde er reichlicher und schärfer. Seiner Natur nach gehöre er zu den laugenhaften Dingen, das alkalinische aber verringere eher den Appetit, Säure sei der Lust zum Essen viel mehr angemessen. Haller nimmt daher an, der Magensaft nehme durch längeres Verweilen Schärfe an und könne so den Appetit vermindern, also das Bedürfnis nach Nahrung einschränken. Ganz zum Schluß fügt er kurz den Hinweis bei, daß von anderen der Hunger als «Ruf der Seele» gedeutet worden sei, bei dem nichts Mechanisches stattfinde.

$\mathrm{Da} \beta$ die Intensität des Hungergefühles von allerlei Faktoren abhängt, erwähnt HaLler gleichfalls: körperliche Arbeit, besonders bei kalter Witterung erhöht den Hunger, ebenso Wurmerkrankungen oder ein erschlaffter Sphincter pylori wegen zu schneller Entleerung des Magens; auch saure Substanzen unterhalten das Hungergefühl, endlich kann dieses durch unterschiedliche Empfindlichkeit der Nerven vergrößert oder vermindert werden.

Diese Ansichten HaLLERs haben noch lange nachgewirkt, zunächst in den späteren Ausgaben seines Grundrisses der Physiologie. Die von Megkel besorgte Auflage aus dem Jahre 1788 folgt in allem Hallers Meinung, 
einzig die Ursache des Hungergefühles wird etwas anders dargestellt, sie sei weniger dem Reiben zuzuschreiben, sondern rühre eher vom Magensafte her, jedoch nicht von dessen Schärfe, sondern von seiner auflösenden Kraft (S. 484 Anm. e). Dieser Meinung schloß sich auch Leveling in seiner Ausgabe von HaLlers Grundriß vom Jahre 1796 an. Die Wirkung der gegenseitig sich reibenden Magenwände ließ auch er nicht mehr gelten, der Hunger komme von der auflösenden Kraft des Magensaftes, der in Mengen sezerniert und nicht verbraucht werde (S. 567).

Auch Johann Friedrich Blumenbach kommt in seinen Institutiones physiologicae von 1798 nicht weiter. Über das Hungergefühl referiert er nur die Meinungen der Autoren, indem er als mögliche Ursachen anführt (S. 258): Reibung der Schleimhautfalten und größere Menge und Schärfe des Magensaftes, erst 1821 fügte er auch noch das Leeregefühl des Magens bei (S.283), einen Faktor, den er offensichtlich von Burdach übernahm (vgl. S. 38). Neu ist bei Blumenbach (S. 259), daß er außer den Unterschieden der Lebensalter und des Körperwuchses als sehr wichtigen Faktor die Eßgewohnheiten nennt, um das Auftreten des Hungergefühles zu bestimmten Zeiten verständlich zu machen. Ein erwachsener, gesunder Mensch, der seiner Sinne mächtig sei, vermag ohne deutliche Einschränkung seiner Kräfte höchstens einen Tag zu fasten, kaum aber über 8 Tage ohne Schädigung des Lebens (S. 260).

Charles-Louis Dumas hat im 3. Band seiner Principes de Physiologie (1800) etwas selbständigere Meinungen geäußert. Es sei schwierig, die wirklichen Ursachen des Hungergefühles zu kennzeichnen (S. 559), Untersuchungen darüber wären aber auch nicht von wirklichem Nutzen. Aus der Literatur (StaнL) übernimmt er die Definition, Hunger sei auf den Bedarf an Nahrung zu beziehen, Appetit dagegen sei ein Gefühl, das den Hunger wünschenswert mache. Welches auch das Ziel sei, auf das sich der Appetit richte, immer bemerke man leicht die Bedeutung der Gewohnheit (S. 560). Der Hunger richte sich im speziellen auf das eine oder andere Objekt, dessen Wahl im allgemeinen von der Eignung der Nahrungsmittel zur Ernährung des Körpers abhänge. Dieser Umstand aber zerstöre mit einem Schlage alle chemischen, physikalischen und mechanischen Erklärungen, die man dem Hungergefühl habe geben wollen.

Dieselben Meinungen wiederholte Dumas im 4. Band seiner Principes de Physiologie (1803, S. 46-48), er begründete sie dort auch sehr ausführlich. Im Hungergefühl sah er nur eine Modifikation des sensiblen Prinzipes des Nervensystemes, und da dieses direkt mit dem Lymphsystem verbunden 
sei, so müßte man dieselben Ansichten auch für das Lymphsystem gelten lassen; es heißt geradezu: der Hunger bezieht sich vor allem auf das Lymphsystem und erklärt dessen Zustand (S. 58). Bei einem im Hungerversuch gestorbenen Hund schiene es, die absorbierende Kraft der Gefäße hätte begonnen, auf die Substanz der Verdauungsorgane selbst zu wirken, deren innere Oberfläche an einigen Stellen angegriffen gewesen sei. Die absorbierenden Gefäße zeigten sich entblößt, sie bewahrten die Fähigkeit zu absorbieren noch lange nach dem Tode (S. 61).

Auch Karl Friedrich Burdach erweist sich in seinem Werke Die Physiologie (1810) als ein verhältnismäßig selbständiger Denker; der Zeitströmung entsprechend argumentierte er zwar sehr mit naturphilosophischen Begriffen, doch findet sich bei ihm zum erstenmal im Zusammenhang mit Erörterungen über die Nahrungszufuhr der Ausdruck «Gemeingefühl». Der zentrale, noch näher zu erläuternde Satz seiner Ausführungen über den Hunger (Par. 179, S. 298) lautet: «Der Hunger ist das dunkle Gefühl von Mangel an indifferent brennstoffigen Substanzen und hat seinen Sitz in dem Magen; er ist ein Gefühl von Leere und unbeschäftigter Assimilationskraft. » $\mathrm{Zu}$ den brennstoffigen Substanzen gehören Stickstoff, Kohlenstoff und Wasserstoff. Sie heißen in ihrer Gesamtheit Brennstoff oder Phlogiston und bilden den Gegensatz zu Sauerstoff, mit dem sie sich in verschiedener Proportion verbinden (Par. 29, S. 56-59). Die Lokalisation des Hungergefühles im Magen begründet oder erläutert BURDAGH nicht, dagegen hat er für das "Gefühl der Leere» auf den Par. 160 verwiesen, in dem das Gemeingefühl (Coenaesthesis) behandelt ist (S. 260-263); er bezeichnet es als «die Fähigkeit eines Organismus, von seinem Körper und dessen Zustand unterrichtet zu werden ». Dieses Gefühl ließe uns unterscheiden, was zu unserer körperlichen Natur gehöre und was nicht, während uns die Sinnesorgane unseren Körper als Teil der äußeren Natur darstellen. Gemeingefühl ist also Selbstanschauung, jedoch auf der niedrigsten Stufe der Vollendung. Wir fühlen die Existenz der Körperteile, ohne dadurch ihre Natur, Lage, Verbindung usw. zu erkennen. Sitz des Gemeingefühls ist vor allem das Rumpfnervensystem, dessen Zentralpunkt das Ganglion coeliacum sei (S. 253), das seinerseits mit dem N. vagus (und dem N. phrenicus) Verbindung haben soll. Das Gemeingefühl pflanzt sich nur mittelbar bis in das Gehirn fort und bewirkt daher nur dunkle Vorstellungen; wir nehmen damit aber nicht nur unsere körperliche Existenz wahr, sondern auch deren Art: Befinden sich die verschiedenen organischen Tätigkeiten untereinander im normalen Gleichgewicht, so fließen sie in dem Gefühl des Wohlseins zusammen, dagegen ent- 
stehen Schmerz oder Unlust bei verminderter Vollkommenheit unseres körperlichen Zustandes, z. B. durch zu starke oder zu schwache Erregungen. Das unangenehme Gefühl von Leere tritt ein, wenn die Erregung wegen mangelnder oder zu schwacher Reize gering ist. Ein Gefühl von Lust entsteht durch jeden U̇bergang zu größerer Vollkommenheit unseres Zustandes. Nach Par. 178 (S. 297) äußert sich Lust z.B. durch das angenehme Gefühl der Sättigung, wenn uns das Gemeingefühl über die Gegenwart solcher Stoffe belehrt, die den Organismus erhalten und ernähren. Sie sind wegen der ständig erfolgenden Zersetzungen und der Verluste durch die Lungen, die Haut und die Abscheidungen notwendig. Werden Speisen und Getränke nicht in verhältnismäßiger Quantität aufgenommen, so entsteht anfänglich Eßlust und vermehrte Verdauungskraft, späterhin aber werden jene Tätigkeiten geschwächt, wie denn jede zu bedeutende Verminderung der Reize und jede zu lang anhaltende Ruhe Lähmung hervorbringen. Teile des Organismus werden dann aufgelöst und resorbiert, Abmagerung und Tod sind die schließliche Folge. Im Winterschlaf und bei verringerter innerer Lebenstätigkeit können manche Tiere lange Zeit ohne Nahrungsaufnahme bleiben. Menschen, die sich während einer längeren Dauer der Nahrung enthielten, waren meist kränklich, besonders hysterische Frauen, sie lagen im Bett und nahmen etwas Wasser zu sich. Junge starke Personen mit guten Verdauungskräften werden durch Nahrungsmangel mehr betroffen als alte, sie sollen dabei auch früher sterben als diese. Damit die Aufnahme neuer Nahrung nicht versäumt wird und dadurch das Leben in Gefahr käme, unterrichten uns die schmerzhaften Regungen des Gemeingefühls vom Mangel an nahrhaften Stoffen, zugleich wird der Instinkt zur Aufnahme von Speise und Trank geweckt.

Ursache des Hungers ist nach Burdach (Par. 179, S. 298), «daß die Muskelfasern des Magens durch Magensaft, die Saugadern und die Nerventätigkeit, also überhaupt durch sauerstoffige, contrahirende Thätigkeiten zu sehr affizirt werden ». Für diese Theorie sprechen ihm alle Erscheinungen: 1. der Muskel ist brennstoffig, wird also vom Mangel an brennstoffiger Nahrung zuerst betroffen; 2. starke Muskelreize bewirken erhöhten Hunger, vermehrte Muskeltätigkeit verursacht gleichfalls stärkeres Hungergefühl; 3. starke Muskelkraft geht mit vermehrter Eßlust parallel; 4. Hunger wird durch stark brennstoffige (d.h. kalorienreiche) Nahrungsmittel am schnellsten und am vollkommensten gestillt; 5. außer durch Aufnahme brennstoffiger Substanzen wird das Hungergefühl vermindert durch stark erregende Affekte, durch körperliche Nervenreize, durch Alkohol, Opium, 
Tabak usw.; 6. sauerstoffige Substanzen vermehren den Hunger; 7. Folgen zu langen Hungerns wirken sich vornehmlich am Muskelsystem aus: es entsteht Mattigkeit, bittrer Geschmack, Kälte der Hände und Füße, Ohnmacht, Krampf, Blutung und schließlich Tod. Nach BurdacH findet man dann den Magen vertrocknet, eingeschrumpft und entzündet, man soll sogar deutlich Stellen bemerken, wo die Saugadern an der Muskelsubstanz genagt und davon resorbiert haben. Leber und Milz seien mit Blut gefüllt, eine Angabe, die den Mitteilungen der übrigen Autoren widerspricht.

Prochaska, dessen Physiologie oder Lehre von der Natur des Menschen 1820 erschien, lehnt sich wieder weitgehend an HaLLER an, er bevorzugt aber mehr naturwissenschaftliche Deutungen. Ich zitiere aus seinem Werk einige Sätze: Verderbnis und Ausscheidung von Stoffen erfordere Ersatz, der zu gehöriger Zeit und in erforderlichem $\mathrm{Maß}$ geschehen muß, damit der Körper weder Überladung noch Mangel leide (S. 364). Da aber Mensch und Tier mit ihrer inneren Einrichtung unbekannt sind, also Zeit und Maß nicht wissen können und deshalb wohl versäumen würden, den körperlichen Bedürfnissen Genüge zu leisten, so schuf die Natur zwei Gefühle, Hunger und Durst. Bei Auftreten des Hungergefühles ist es Zeit zu essen, die Nahrungsaufnahme verschafft deutliche Lustempfindungen. Tilgung des Hungergefühles zeigt, daß genug aufgenommen wurde; wer dieses Ziel überschreitet, wird mit den Folgen der Überladung bestraft.

Hunger tritt beim erwachsenen gesunden Menschen spätestens 12 Stunden nach einer mäßigen Mahlzeit wieder ein, bei Kindern früher und öfter. Große Unterschiede in der Nahrungszufuhr bestehen auch je nach dem Temperament und der Gewohnheit (S. 365).

Sitz des Hungers (S. 367) ist der Magen; sein Saft wird wie alle anderen schärfer, was den Magen reizt und das Gefühl des Hungers verursacht. Schärfe durch Hunger kommt aber allen Säften des Körpers zu, trotzdem wird sie nur vom Magen empfunden, daher scheint, daß die Magennerven speziell gegen die Schärfe empfindlich sind. Salze, Säuren und Gewürze kommen der Schärfe des Magensaftes zu Hilfe und vermehren die Eßlust, indem sie die Empfindlichkeit der Magennerven anregen.

Magendie zitiere ich nach der 2. Auflage seines Précis élémentaire de Physiologie von 1825. Auch er geht von der Notwendigkeit aus, daß die Verluste des Körpers ersetzt werden müssen, was durch Nahrungsaufnahme geschehe (S. 22). Zu deren Regulierung dienen einige instinktive Gefühle. Bei leerem Magen reizen Hunger und Durst zur Nahrungsaufnahme, gegen die Zufuhr von Nahrungsstoffen wirken das Gefühl der Sättigung und der Wi- 
derwille. Die Hungerempfindung ist nach Art und Intensität bei den einzelnen Menschen sehr verschieden (S. 23), manche essen nur, weil die gewohnheitsmäßige Zeit gekommen ist. Wird das Nahrungsbedürfnis nicht erfüllt, so kann das Hungergefühl geradezu schmerzhaft werden.

Den bis zu seiner Zeit vorgebrachten Meinungen über die lokalen Erscheinungen des Hungers stellt Magendie seine eigenen Beobachtungen gegenüber. Nach 24, 48 und selbst 60 Stunden völliger Nahrungsenthaltung fand er den Magen nie kontrahiert und verengert, erst im Laufe des 4. und 5. Tages schien ihm der Magen kleiner geworden und in seiner Lage etwas verändert (S. 25). Віснат habe gemeint, daß sich die Bauchwand jeweils dem Füllungszustand des Magens entsprechend weiter oder enger stelle. Dieser Ansicht widerspricht MAGENDIE auf Grund eigener Beobachtungen; er meint, daß bei leerem Magen alle übrigen Hohlorgane des Bauchraumes sich leichter ausdehnen können, daher sei z.B. die Gallenblase im Hungerzustand so stark gefüllt. Rückfluß von Galle in den leeren Magen ist aber kein normaler Befund (S.26), kann also für die Ursache des Hungergefühles nicht in Frage kommen.

Wie Dumas fand Magendie mit längerer Fastendauer immer weniger Schleim im Magen, dessen Wand wie die aller übrigen Bauchorgane im Hungerzustand weniger Blut erhält (S. 27).

$\mathrm{Zu}$ den allgemeinen Hungererscheinungen zählt Magendie die zunehmende Schwäche und Abnahme der Tätigkeit aller Organe; die Zirkulation und die Atmung seien verlangsamt, die Körpertemperatur sinkt, alle Arten von Sekret gehen zurück, einzig die Absorption soll erhöht sein, was aber nicht zuverlässig erwiesen sei. Zunahme des Hungergefühls tritt auch nach kalten Bädern und trockener Reibung der Haut ein, Abnahme des Hungergefühles verursachen neben den äußeren Lebensumständen gewisse Substanzen wie Opium und warme Getränke.

Alle die verschiedenen früher genannten Mechanismen, die das Hungergefühl auslösen sollen, lehnt MAGendie als Ursache desselben ab (S. 29). Hunger entsteht, wie alle inneren Empfindungen, durch die Tätigkeit des Nervensystemes und unterliegt damit den allgemeinen Gesetzen der Organisation. Zum Schluß wendet sich Magendie gegen die Ausdehnung des Begriffes Hunger auf die schließlich zum Tode führende Nahrungsenthaltung, er sähe den Begriff Hunger lieber beschränkt auf den eingangs definierten physiologischen Instinkt (S. 30).

Rudolphi faßte in seinem Grundriß der Physiologie 1828 zusammen, was in den bisherigen Veröffentlichungen referiert wurde. Stärker als alle an- 
deren Autoren betont er die individuellen Unterschiede in der Empfindung des Hungergefühles, das er nur anfänglich im Magen lokalisiert, später soll es sich weiter ausbreiten (S. 5-6). Wie nicht anders zu erwarten, sind die Vorstellungen oft mehr naturphilosophisch als naturwissenschaftlich; z.B. heißt es: Man darf wohl nur im allgemeinen an die verschiedene Nervenstimmung des Magens bei Leere und Untätigkeit denken, beides Zustände, die schon BurdacH mit dem Hungergefühl in Verbindung gebracht hatte. Schwächliche Menschen empfinden etwa gleich Übelkeit und Kopfschmerz, werden wohl auch ohnmächtig, wenn sie etwas länger als gewöhnlich nüchtern bleiben. Als kritisch erweist sich RudolPHI durch die Feststellung, daß angebliches gänzliches Fasten bei frisch und gesund aussehenden Menschen von einiger Körperkraft für Betrug gehalten werden muß (S. 12-13).

Nur berichtend ist auch, was JoHANnes MüLLER in seinem Handbuch der Physiologie des Menschen (1833) mitteilt. Auf S. 465/6 heißt es: «Die örtlichen Empfindungen des Hungers, welche sich auf die Verdauungswege beschränken und im N. vagus ihren Sitz zu haben scheinen, sind Gefühle von Druck, Bewegung, Zusammenziehung, von Übelkeit mit Kollern, später Schmerzen.» Abgelehnt wird, daß als Ursache dieser Empfindungen der Speichel, die Galle, eine Reibung der Magenwände und Schärfe des Magensaftes anzusehen seien. Die Nahrungsmittel seien für die Verdauungsorgane adäquate Reize; fehlen diese, so bringen die Nerven den Zustand des Organes zum Bewußtsein. Möglicherweise könnten nach Durchschneidung des $\mathrm{N}$. vagus die örtlichen Empfindungen des Hungers, des Appetites und der Sättigung fehlen. Aufgehoben wird die Empfindung des Hungers 1. durch eine von der aufgenommenen Nahrung ausgelöste Veränderung der Nerven des Magens, 2. durch stärkere Empfindungen und Tätigkeiten, die das Sensorium in Leidenschaften oder Meditationen beschäftigen, und 3. durch Änderung des Sensoriums selbst z.B. durch Opium. Diese Alternation des Sensoriums mache vielleicht auch das häufige Fasten bei Irren verständlich, die möglicherweise keine örtlichen Empfindungen des Hungers mehr haben. Nur die allgemeinen Folgen des Fastens sind nach JoH. MüLLER unter ungleichen Zuständen der Verdauungsorgane meist gleich, nämlich die allgemeine Hinfälligkeit, die ständig zunehmende Kraftlosigkeit, Abmagerung, Fieber, Irrereden und Erregungszustände wechselnd mit tiefster Niedergeschlagenheit. Der Atem wird stinkend, der Harn scharf, der Magen kontrahiert sich, die Gallenabsonderung geht zurück, sie hört jedoch nicht völlig auf; die Schleimbildung verringert sich, Milch, Speichel und andere Sekrete fließen kaum mehr. Daß völlige Nahrungsenthaltung zum Tode 
führt, wird gleichfalls angegeben, wobei die großen Unterschiede in der Empfindlichkeit verschiedener Tierarten betont werden. «Menschen ertragen Hunger und Durst in der Regel nicht länger als eine Woche, den bloßen Hunger viel länger, in Krankheiten noch länger, besonders Irre.»

Sehr ausführlich ist die Darstellung, die TiedemanN (1836) in seiner Physiologie des Menschen gab. Er definierte in einem Abschnitt «Vom Nahrungstrieb» den Hunger als «Begierde nach festen Nahrungsmitteln » (S. 21) und unterschied drei Grade: Eßlust, Eßbegierde und Hunger (S. 23). Der Hunger wird als eines der schmerzhaftesten und qualvollsten Gefühle bezeichnet, er erfülle die Seele mit Unruhe, peinigender Angst und Verzweiflung. Auch Tiedemann hält noch daran fest, daß durch den Nahrungstrieb Tätigkeit und Wirken des Menschen erzwungen werden. Bei ablenkender Beschäftigung oder in angenehmer Gesellschaft kann sich das zu gewohnter Stunde auftretende Hungergefühl verlieren, ohne befriedigt worden zu sein.

In extenso berichtet Tiedemann dann über Beobachtungen an hungernden Tieren (S. 26-31). Ich übergehe die Einzelheiten dieser Befunde, weil sie sich bei der Beschreibung der Auswirkung des Hungerzustandes auf den menschlichen Körper wiederholen (S. 32-36). Nach 1- bis 2 tägigem Fasten ist beim Menschen die Schleimhaut der Mund- und Rachenhöhle trocken, etwas gerötet, geschwollen und bei Berührung schmerzhaft. Außer dem Gefühl der Leere, des Spannens und Ziehens in der Magengegend stellen sich oft Übelkeit und Magenkrämpfe ein. Die Menge des Blutes nimmt ab, verringert ist auch die Zahl der abgeschwächten Pulsschläge. Als Folge verminderter Wärmebildung tritt Frösteln ein, die Haut wird wegen der schlechten Blutversorgung blaß und welk, die Gesichtszüge werden scharf und hager; das Fettgewebe und die Muskeln und damit auch das Körpergewicht nehmen ab. Mit der verringerten Blutmenge geht auch die Abscheidung aller Sekrete zurück, der Speichel wird klebrig, die Haut wegen geringerer Ausdünstung trocken, die ausgeatmete Luft wird übelriechend, ebenso der an Menge reduzierte Harn. Die Atmung ist beschleunigt und ungleich, Gähnen ist häufig, Schlaflosigkeit ist bei Hungrigen die Regel. Die Tätigkeit der Sinne wird abgestumpft, die Aufmerksamkeit, das Gedächtnis und die Urteilskraft lassen nach, Unlust und Mißmut sind die Folge. Der Selbsterhaltungstrieb äußert sich bei Hungernden in gröbstem Egoismus; fehlt es an Mitteln, diesen Trieb zu befriedigen, so tritt schließlich unter Irrereden, Krämpfen und Ohnmachtsanfällen der Tod ein. Daß der menschliche Geist imstande ist, den Selbsterhaltungstrieb zu überwinden, beweisen die immer wieder vorkommenden Fälle freiwilligen Fastens 
bis zum Tode, wofür auch Tiedemann einige Beispiele anführt (S. 36-38). An Leichen Verhungerter sind die hochgradige Abmagerung, die Rückbildung der Muskeln, der völlig kontrahierte Magen und die weitgehende Leere des Blutgefäßsystems die deutlichsten anatomischen Befunde (S. 38).

Den Ursachen des Hungergefühles widmete TiedemanN ein besonderes Kapitel (S. 42): Wie Magendie lehnte er die von den früheren Autoren entwickelten mechanischen und chemischen Vorstellungen ab. Tatsächlich ist der Hunger nicht als eine durch äußere Einwirkungen oder Reize entstandene Empfindung zu deuten, er ist vielmehr ein inneres Gefühl eigener Art, «das aus einer Veränderung des Zustandes und der vitalen Stimmung des Nervensystemes beim Bedürfnis nach Nahrungsmitteln entspringt» (S. 44). Anfänglich auf die Nerven des Magens beschränkt, leidet mit der Verminderung der Blutmenge die Ernährung des ganzen Körpers und damit auch die des Nervensystemes, dessen Vitalität modifiziert wird, daraus geht dann das Hungergefühl hervor. Örtlich handelt es sich bei dem Gefühl der Eßlust und des Nüchternseins zunächst um eine gesteigerte Empfindlichkeit oder erhöhte Reizbarkeit der Nerven «in Folge des Leerseyns des Magens und seiner nicht statthabenden Verrichtung» (S. 44), von Zuständen also, die genau so von BurdacH angeführt wurden, doch ist sein Name in diesem Zusammenhang nicht genannt. Die eigentlichen Veränderungen, die am peripheren und zentralen Nervensystem durch den Hungerzustand eintreten, sind ebensowenig bekannt wie die Vorgänge im Gehirn bei anderen Gefühlen. Daß aber tatsächlich gesteigerte Empfindlichkeit der Magennerven für das Hungergefühl bedeutsam ist ergibt sich aus der Wirkung einiger Substanzen, die die Nervenreizbarkeit herabsetzen; Opium, Belladonna, Kirschlorbeerwasser, Rauchen und Kauen von Tabak, Öl und warmes Wasser vermindern das Hungergefühl (S. 48).

Da alle diese Befunde durch ausführliche Zitate belegt sind, wird Tiedemanns Arbeit zu der bestdokumentierten seit Hallers Elementa physiologiae. So nimmt es nicht wunder, daß Valentin sich in seinem Lehrbuch der Physiologie des Menschen (2.Auflage, 1847) weitgehend auf Tiedemann stützt. Da der Hunger von einer subjektiven Tätigkeit der Nerven ausgeht, so beherrschen ihn auch dieselben Gesetze, die viele andere Erscheinungen des Nervensystemes bestimmen (S. 230). Zufuhr von Nahrungsstoffen durch Klistiere vermindert das Hungergefühl bei Menschen, die am Schlucken behindert sind (S. 231). Ablenkung vom Hungergefühl durch Tätigkeit ist möglich, andererseits wird der Untätige heftiger an seine Nahrungsbedürfnisse gemahnt. Narkotische Mittel verringern die Nervenerregung, aus der 
das Hungergefühl hervorgeht (S. 231). Umgekehrt wird zum Teil neu angeführt, daß örtlich wirkende Reizmittel, wie Pfeffer, Ingwer und andere Gewürze, weingeistige Getränke, manche Salzverbindungen sowie bittere Pflanzenextrakte den Trieb zur Nahrungsaufnahme vergrößern (S. 231).

Die Ursache des Hungergefühles ist nach Valentin ebenso dunkel wie, die der Tastempfindung, beide erscheinen bis zu einem gewissen Grade vergleichbar, insbesondere verwandeln beide übermäßige Reize in Schmerzempfindung (S. 232). Hier liegt wieder eine Annäherung an BuRdachs «Gemeingefühl» vor, ohne daß jedoch diese Bezeichnung gebraucht wird. Die mechanischen und chemischen Vorstellungen, die sich ältere Forscher von den Ursachen des Hungers bildeten, widerstreiten nach Valentis den Tatsachen, «sie gehören nur noch der Geschichte der Wissenschaften an» (S. 232).

Während etwa zwei Dritteln der hier behandelten Zeitspanne beherrschten Hallers Meinungen die lehrbuchmäßige Darstellung des Hungers. Die Bemühungen, aus mehr oder weniger fundierten Beobachtungen Aufklärung über dieses Gefühl zu erlangen, mußten indessen bei dem damaligen Wissensstande scheitern, da bei einer so stark psychisch beeinflußten Empfindung wie dem Hungergefühl aus Tierversuchen keine Erkenntnis zu gewinnen war. So wurde BuRdachs Idee vom Gemeingefühl zu einem bis heute erhalten gebliebenen Begriff, wennschon wir ihm nicht mehr die Ausweitung geben, die er bei BURDACH fand - sie stand hier auch nicht zur Diskussion. Im letzten Drittel der vorstehend studierten Epoche bekannten sich mehr und mehr Autoren - ohne ausdrücklich BURDAGH zu nennen - zu seiner Meinung und brachten unter verschiedener Bezeichnung das gleiche wie er zum Ausdruck (Magendie, Joh. Müller, Tiedemann, Valentin). 


\section{Literaturnachweis}

Blumenbach Jo. Frid., Institutiones physiologicae, Gottingae 1798, 4. ed. 1821.

Burdach Karl Friedrich, Die Physiologie, Leipzig 1810.

Dumas Charles-Louis, Principes de physiologie, Tom. 3 et 4, Paris 1800/1803.

Haller A. Primae lineae physiologiae in usum praelectionum academicarum, Gottingae 1747.

Haller A. von, Elementa physiologiae corporis humani, Vol. 6, Bernae 1764.

Leveling Heinrich Maria von, Albert's von Haller Grundriß der Physiologie für Vorlesungen, Erlangen 1795.

Magendie F., Précis élémientaire de Physiologie, $2^{\mathrm{e}}$ éd., Paris 1825.

Meckel P. F., Alberts von Haller Grundriß der Physiologie für Vorlesungen, Berlin 1788.

Müller Johannes, Handbuch der Physiologie des Menschen, 1. Band, Coblenz 1833.

Prochaska Georg, Physiologie oder Lehre von der Natur des Menschen, Wien 1820.

Rudolphi Karl Asmund, Grundriß der Physiologie, 2. Band, 2. Abt. Berlin 1828.

Tiedemann Friedrich, Physiologie des Menschen. 3. Band, Darmstadt 1836.

Valentin G., Lehrbuch der Physiologie des Menschen, 2. Auflage, 1. Band, Braunschweig 1847. 